\title{
Structural study of Clopamide drug and copper (II) complexes under different crystallization conditions
}

\author{
G. Tamás Gál, Nóra V. May, Petra Bombicz \\ Centre for Structural Science, Research Centre for Natural Sciences, Magyar tudósok körútja 2. H-1117 Budapest, Hungary \\ may.nora@ttk.hu
}

Knowledge on conformation and crystal structures (including crystal polymorphs and solvatomorphs) is important in the use and development of active pharmaceutical ingredients (API). Polymorphism plays a very important role in the bioavailability of a drug [1] as the physico-chemical properties (e.g. solubility, stability) of polymorphs can differ significantly [2]. Clopamide (4-chloro-N-2,6dimethylpiperidin-1-yl)-3-sulfamoylbenzamide) drug is used worldwide in the treatment of hypertension and oedema and despite its medical application the crystal structure has not yet been investigated. It has also been shown that some diuretic may also cause urinary loss of certain trace elements or modify their levels in blood and may induce changes in the levels of copper in normal hypertensives [3]. According to the molecular structure it is noticeable that the carbonyl oxygen and the piperidine nitrogen of Clopamide are able to coordinate to metal centres, so the complexation of Clopamide with metal ions in the human body may be responsible for this side effect (Fig. 1). To gain a better insight into the structure and interaction of this drug molecule with metal ions, structural study of Clopamide compound and its coordination compounds with copper(II) have been studied under different crystallization conditions. The crystal structure of this drug and its copper(II) complexes were studied by screening different solvatomorph and polymorph crystals and the structures were determined by single-crystal X-ray diffraction. Our challenge was to detect the conformations and possible arrangements of the complexes induced by coordination bond and by different secondary interactions. These investigations enrich the knowledge on the aspects which contribute to the development of materials with specific properties. The crystal structures of anhydrous, and hemihydrate form of Clopamide have been determined. We present how the inclusion of water contributes to the crystal perfection of the drug crystals. The newly defined chalcogen bonds are recognised in the Clopamide anhydrate crystals being in competition with intramolecular halogen bonds. The bis-ligand copper(II) complex crystals were synthesized from the homolouges series of alcohols, with the inclusion of solvent molecules, resulting four isostructural [4] crystals with increasing size of void and unit cell volumes in the order of $\mathrm{MeOH}, \mathrm{EtOH}, \mathrm{PrOH}$ and iPrOH. From organic solvents, three solvent free polymorphs and a crystal containing dichloromethane was prepared. All $\mathrm{Cu}$ (II) compounds are of square-planar geometry, in which copper(II) centres are coordinated by piperidine-N and carbonyl-O donor atoms in a five-membered chelate ring with the two ligands in trans positions. The 2,6-dimethylpiperidine units of the molecule is perpendicular to the plane of the coordination sphere, preventing axial coordination of solvate molecules. The solution structure of the copper(II) complex have been investigated in DMSO by EPR spectroscopy.

(a)

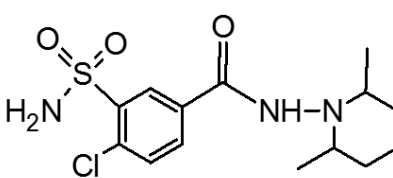

(b)

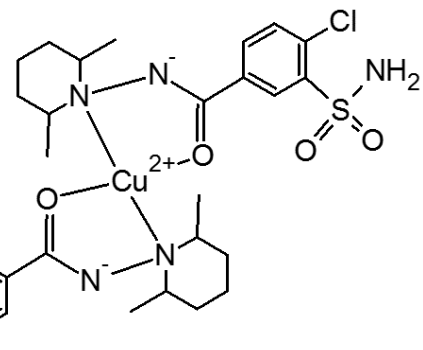

Figure 1 General formula of (a) Clopamide (LH) and (b) its bis-ligand copper(II) complex.

[1] Censi, R. \& Di Martino, P. (2015) Molecules 20 18759-18776.

[2] Chemburkar, S. R., Bauer, J., Deming, K., Spiwek, H., Patel, K., Morris, J., Henry, R., Spanton, S., Dziki, W., Porter, W., Quick, J., Bauer, P., Donaubauer, J., Narayanan, B. A., Soldani, M., Riley, D., McFarland, K. (2000) Org. Process Res. Dev. $4413-417$

[3] Du Preez, M., \& Lockett, C. J. (1991) J. Am. Coll. Nutr. 10, 34-37.

[4] Bombicz, P. (2017) Crystallogr. Rev. 23, 118-151.

Keywords: Clopamide, solvatomorphs, polymorphs, isostructural, copper(II) complexes

The support of the National Research, Development and Innovation Office - NKFIH through OTKA KH129588 and K124544 are gratefully acknowledged.

Acta Cryst. (2021), A77, C1199 